\title{
General Lower Bounds for Resonances in One Dimension*
}

\author{
Evans M. Harrell II \\ Department of Mathematics, The Johns Hopkins University, Baltimore, MD 21218, USA
}

Abstract. Lower bounds are derived for the magnitude of the imaginary parts of the resonance eigenvalues of a Schrödinger operator

$$
-\frac{d^{2}}{d x^{2}}+V(x)
$$

on the line, depending only on the support and bounds of $V$ and on the real part of the resonance eigenvalue. For example, if the resonance eigenvalue is denoted $E+i \varepsilon$, then there exist $C$ and $\ell_{0}$ depending only on $\|V\|_{\infty}$ and $E$ such that if the support of $V$ is contained in an interval of length $\ell>\ell_{0}$, then

$$
|\varepsilon|>\frac{m^{3} \sqrt{E}}{(m+\sqrt{E})^{2}} \exp (-m \ell)\left(1-C \ell^{-1}\right),
$$

where $m=\|V(x)-E\|_{\infty}^{1 / 2}$.

Spencer has recently raised the question of whether there is a lower bound for the magnitude of the imaginary parts of the resonance eigenvalues for a quantummechanical particle in a compactly supported potential that is randomly generated, and therefore neither necessarily regular nor known at all in detail [1]. The purpose of this note is to derive such lower bounds in the one-dimensional case assuming knowledge of the real part. The many somewhat distinct notions of resonance for operators $-\Delta+V(x)$ on $L^{2}\left(\mathbb{R}^{n}\right)$ become all more or less the same if $n=1$ and $V$ is compactly supported. A physically motivated definition would be a complex value of $k$ for which a scattering state for

$$
\frac{-d^{2}}{d x^{2}}+V(x)
$$

* Research supported by NSF grant MCS 7926408 
proportional to $\exp (-i k x)$, when $x<\inf \operatorname{supp} V$ is proportional to $\exp (+i k x)$ when $x>\sup \operatorname{supp} V$. It may as well be assumed that $\operatorname{supp} V \subset[0, l]$. Physical tunneling arguments lead one to expect that if $l$ is large, then the resonance width should be at least on the order of $\exp (-C(V, E) l)$, where $C$ depends on $V$ and the energy $E$ in some simple way. This is essentially what is shown in Eq. (8). The assumption about the support of $V$ makes the resonance equivalent to an eigenvalue of a nonself-adjoint Sturm-Liouville problem on a finite interval:

Definition. Let $V$ be a bounded, real-valued, measurable function of $x \in[0, l]$ and $\psi$ be a function (with absolutely continuous first derivative) satisfying

$$
-\psi^{\prime \prime}+\left(V-k^{2}\right) \psi=0
$$

a.e. and

$$
\psi^{\prime}(0) / \psi(0)=-i k, \quad \psi^{\prime}(l) / \psi(l)=+i k,
$$

$0>\arg k>-\pi / 4$. Then $k^{2}$ is called a resonance eigenvalue for $\frac{-d^{2}}{d x^{2}}+V$ and $\left|\operatorname{Im} k^{2}\right|$ is called the resonance width.

\section{Remarks}

1. By scaling the coordinate one could assume $l=1$ or alternatively $\|V\|_{\infty} \equiv \sup _{0 \leqq x \leqq l}|V(x)|=1$, but for physical applications the limits $l \rightarrow \infty$ or $\|V\|_{\infty} \rightarrow \infty$ or some combination of these can be of interest, so the dependence on these quantities will be left explicit. A limit related to those was studied in [2], where a detailed perturbation and resonance theory was worked out (on a half-line) for potentials with certain regularity assumptions, especially at the boundary between the region where $V<0$ and the confining barrier where $V>0$. In that case detailed uniform estimates of $\psi$ can be resorted to to obtain precise formulae. Moreover, the existence of shape resonances was proved in limiting cases, whereas the present article assumes their existence. An arbitrarily chosen $V$ could quite easily not have any resonances. Discussions of quantum resonances from various points of view can be read in [3-5].

2. On physical grounds one might expect the potential producing the narrowest resonance width to have roughly the form

$$
V(x)=\left\{\begin{array}{l}
V_{\max }, 0 \leqq x \leqq a, l-a \leqq x \leqq l \\
V_{\min }, a<x<l-a
\end{array}\right.
$$

for some optimally chosen $a$. These resonance widths can be worked out by hand and are somwhat larger than Eqs. (6) and (7). Also, many obvious small improvements could be made in the bounds Eqs. (6) and (7) at the expense of some complication, by modifying the proof. There may thus be a little room for improvement, although the general functional form of these bounds is surely optimal. The lower bounds on $\left|\operatorname{Im} k^{2}\right|$ will be derived by expressing it in terms of $\psi$ and estimating $\psi$. 
Lemma 1. Let $\operatorname{Re} k^{2}=E$ and $\operatorname{Im} k^{2}=\varepsilon$. Then

$$
\varepsilon=-\frac{\operatorname{Re} k\left(|\psi(0)|^{2}+|\psi(l)|^{2}\right)}{\int_{0}^{l}|\psi(x)|^{2} d x}
$$

Proof. This comes from integration by parts, and is only a variant of a formula used in [2] and elsewhere. In particular, one finds from

$$
2 i \varepsilon \int_{0}^{l}|\psi(x)|^{2} d x=\int_{0}^{l}\left\{\bar{\psi}(x)\left[-\frac{d^{2}}{d x^{2}}+V-E\right] \psi(x)-\psi(x)\left[-\frac{d^{2}}{d x^{2}}+V-E\right] \bar{\psi}(x)\right\} d x
$$

that

$$
\begin{aligned}
\varepsilon & =\frac{\operatorname{Im}\left[\psi(l) \bar{\psi}^{\prime}(l)-\psi(0) \bar{\psi}^{\prime}(0)\right]}{\int_{0}^{l}|\psi(x)|^{2} d x} \\
& =-\frac{\operatorname{Re} k\left[|\psi(0)|^{2}+|\psi(l)|^{2}\right]}{\int_{0}^{l}|\psi(x)|^{2} d x}
\end{aligned}
$$

because of the boundary conditions (2).

Since the eigenvalue equation is linear it may be supposed that

$$
1=\psi(0) \geqq|\psi(l)|,
$$

if necessary by changing variables to $x^{\prime}=l-x$. The problem is thus to bound the denominator of (3) above. This is easy to do with standard comparison arguments, which are best used in the framework of integral equations to avoid the problem of discontinuities in $V$ and therefore $\psi^{\prime \prime}$.

Lemma 2. For $0<x \leqq l$,

$$
|\psi(x)|<\sqrt{1+\left((\operatorname{Im} k)^{2}+(\operatorname{Re} k)^{2}\right) x^{2}} \exp \left(\int_{0}^{x}\left|V\left(x^{\prime}\right)-E-i \varepsilon\right|\left(x-x^{\prime}\right) d x^{\prime}\right)
$$

and

$$
\begin{aligned}
|\psi(x)| & <\cosh \left(m_{x} x\right)+\frac{|\operatorname{Im} k|+\operatorname{Re} k}{m_{x}} \sinh \left(m_{x} x\right) \\
& <\left(1+\frac{|\operatorname{Im} k|+\operatorname{Re} k}{m_{x}}\right) \exp \left(m_{x} x\right),
\end{aligned}
$$

where $m_{x}=\sup _{0 \leqq y \leqq x} \sqrt{|V(y)-E-i \varepsilon|}$.

For the interesting physical limits $l \rightarrow \infty$ or $|V(x)|$ large, (5) is much the better $\left(0\left(l e^{c l}\right)\right.$ versus $\left.0\left(l e^{c l^{2}}\right)\right)$ and is of the right general form for quantum-mechanical tunneling. The former formula (4) might be better under other circumstances. 
Proof. Since integration of (1) with (2) yields the integral equation

$$
\begin{gathered}
\psi(x)=1-i k x+\int_{0}^{x} d x_{1} \int_{0}^{x_{1}} d x_{2}\left(V\left(x_{2}\right)-E-i \varepsilon\right) \psi\left(x_{2}\right), \\
|\psi(x)| \leqq \sqrt{1+\left((\operatorname{Im} k)^{2}+(\operatorname{Re} k)^{2}\right) x^{2}}+\int_{0}^{x}|V(y)-E-i \varepsilon|(x-y)|\psi(y)| d y,
\end{gathered}
$$

Eq. (4) is just Gronwall'y inequality $[6,7]$. The inequality is strict because it is strict near $x=0$. For Eq. (5), observe that

$$
\begin{aligned}
|\psi(x)| & \leqq \sqrt{(1+\operatorname{Im} k x)^{2}+(\operatorname{Re} k)^{2} x^{2}}+\int_{0}^{x} d x_{1} \int_{0}^{x_{2}} d x_{2} m_{x}^{2}\left|\psi\left(x_{2}\right)\right| \\
& \leqq 1+(|\operatorname{Im} k|+\operatorname{Re} k) x+\int_{0}^{x} d x_{1} \int_{0}^{x_{2}} d x_{2} m_{x}^{2}\left|\psi\left(x_{2}\right)\right|
\end{aligned}
$$

Now for $x \leqq z$,

$$
f(x) \equiv \cosh \left(m_{z} x\right)+\frac{|\operatorname{Im} k|+\operatorname{Re} k}{m_{z}} \sinh \left(m_{z} x\right)>0
$$

is just the solution of

$$
f(x)=1+(|\operatorname{Im} k|+\operatorname{Re} k) x+\int_{0}^{x} d x_{1} \int_{0}^{x_{2}} d x_{2} m_{z}^{2} f\left(x_{2}\right) .
$$

By continuity $f(x)>|\psi(x)|$ in some neighborhood of $x=0$, and it is then clearly impossible for $f\left(x_{0}\right)-\left|\psi\left(x_{0}\right)\right|$ to vanish for any positive $x_{0}$, as from the integral equations $f\left(x_{0}\right)-\left|\psi\left(x_{0}\right)\right|$ is the sum of a nonnegative function and the integral of a nontrivial nonnegative function.

Theorem 3. For any $\delta>0$,

$$
|\varepsilon| \geqq \min \left(\delta, \frac{(\operatorname{Re} k) \exp \left(-\frac{1}{4} m^{2}\left(1+\delta^{2} / m^{4}\right)^{1 / 2} l^{2}\right)}{l+\left(\left(\frac{\delta}{2 \operatorname{Re} k}\right)^{2}+(\operatorname{Re} k)^{2}\right) \frac{l^{3}}{12}}\right)
$$

and

$$
|\varepsilon| \geqq \min \left(\delta, \frac{(\operatorname{Re} k)\left(\exp \left(m\left(1+\delta^{2} / m^{4}\right)^{1 / 4} l\right)-1\right)^{-1}}{\frac{1}{m}\left(1+\frac{\delta}{2 m \operatorname{Re} k}+\frac{\operatorname{Re} k}{m}\right)^{2}}\right),
$$

where $m=\sup _{0 \leqq x \leqq l} \sqrt{|V(x)-E|}$.

Formula (7) will ordinarily be stronger than Formula (6). Note that these are equivalent to bounds involving $E$ alone or $\operatorname{Re} k$ alone because

$$
\sqrt{E}=\sqrt{(\operatorname{Re} k)^{2}-(\operatorname{Im} k)^{2}}<\operatorname{Re} k<\sqrt{E+\varepsilon^{2} / 4 E}
$$


Corollary 4. For any $\eta>0$, there exists $l_{0}$, depending only on $\|V\|_{\infty}$ and $E$, such that if $l>l_{0}$, then

$$
|\varepsilon|>\frac{m^{3} \sqrt{E}}{(m+\sqrt{E})^{2}}(1-\eta) \exp (-m l) .
$$

The number $\eta$ may be chosen less than a constant over $\ell$.

Proof. The theorem comes from substituting the bounds of Lemma 2 into the formula of Lemma 1, taking into account that if $x>l / 2$ it is more efficient to replace Lemma 2 with the analogous bounds for the interval $[l / 2, l]$. Thus, for instance, from (4),

$$
\begin{aligned}
\int_{0}^{l / 2}|\psi(x)|^{2} d x \leqq & \exp \left(2 \int_{0}^{l / 2}\left|V\left(x^{\prime}\right)-E-i \varepsilon\right|\left(l / 2-x^{\prime}\right) d x^{\prime}\right) \\
& \cdot\left(\frac{l}{2}+\left((\operatorname{Im} k)^{2}(\operatorname{Re} k)^{2}\right) \frac{l^{3}}{24}\right) \\
\leqq & \frac{1}{2}\left(l+\left((\operatorname{Im} k)^{2}+(\operatorname{Re} k)^{2}\right) \frac{l^{3}}{12}\right) \exp \left(2 m_{l / 2}^{2}\left(\frac{l^{2}}{8}\right)\right)
\end{aligned}
$$

Since $|\operatorname{Im} k|=\left|\frac{\varepsilon}{2 \operatorname{Re} k}\right|$, which is assumed $<\frac{\delta}{2 \operatorname{Re} k}$ for the inequality (6), and $m_{l / 2}^{2} \leqq m_{l}^{2}=m^{2}\left(1+\varepsilon^{2} / m^{4}\right)^{1 / 2},(6)$ follows. Inequality (7) is similar, and the corollary follows immediately from it, with a simple expansion of the exponent.

In closing, note that in the $n$-dimensional case one can of course integrate by parts and estimate eigenfunctions with comparison theorems, but what is lacking is the easy control on the gradient and value of the resonance wave-function at the edge of the support of $V$.

\section{References}

1. Simon, B.: Private communication

2. Ashbaugh, M.S., Harrell, E.M.: Perturbation theory for shape resonance and large barrier potentials. Commun. Math. Phys. 83, 151-170 (1982)

3. Reed, M., Simon, B.: Methods of modern mathematical physics. IV. Analysis of operators. New York: Academic Press 1978

4. Lavine, R.: Spectral density and sojourn times. In: Atomic scattering theory, Nuttall, J. (ed.). London, Ontario: University of Western Ontario Press 1978

5. Newton, R.G.: Scattering theory of waves and particles. New York: McGraw-Hill 1966

6. Hartman, P.: Ordinary differential equations. New York: Wiley 1964

7. Reid, W.T.: Ordinary differential equations. New York: Wiley 1971; Properties of solutions of an infinite system of ordinary differential equations of the first order with auxiliary boundary conditions. Trans. Am. Math. Soc. 32, 284-318 (1930). In particular, this last article states Gronwall's inequality assuming only that the function multiplying the solution within the integral is nonnegative and integrable

Communicated by T. Spencer

Received March 8, 1982 
